\title{
Review of: "Automated characterisation of microglia in ageing mice using image processing and supervised machine learning algorithms"
}

Shuohong Wang ${ }^{1}$

1 Harvard University

Potential competing interests: The author(s) declared that no potential competing interests exist.

This paper proposes an automated characterization system for microglia in ageing mice using computer vision techniques and support vector machine (SVM), which is the first of its kind to analyze such large quantities of microglia (entire retinas) with such morphological detail. The area under the ROC curve > 0.99 , which demonstrates the efficacy of the trained SVM. The findings may suggest that senescent changes in the microglia could contribute towards AD pathology.

BTW, I'm concerning about the figures in the manuscript. The figures have a really low resolution in the pdf version I downloaded from Scientific Reports website. 